\title{
Weakly strongly star-Menger spaces
}

\author{
GauraV Kumar ${ }^{1}$ \\ BriJ K. TYAGI ${ }^{2}$ \\ 1 Department of Mathematics, University \\ of Delhi, New Delhi-11000\%, India. \\ gaurav.maths.du@gmail.com \\ 2 Atma Ram Sanatan Dharma College, \\ University of Delhi, New Delhi-110021, \\ India. \\ brijkishore.tyagi@gmail.com
}

\begin{abstract}
A space $X$ is called weakly strongly star-Menger space if for each sequence $\left(\mathcal{U}_{n}: n \in \omega\right)$ of open covers of $X$, there is a sequence $\left(F_{n}: n \in \omega\right)$ of finite subsets of $X$ such that $\overline{\bigcup_{n \in \omega} S t\left(F_{n}, \mathcal{U}_{n}\right)}$ is $X$. In this paper, we investigate the relationship of weakly strongly star-Menger spaces with other related spaces. It is shown that a Hausdorff paracompact weakly star Menger $P$-space is star-Menger. We also study the images and preimages of weakly strongly star-Menger spaces under various type of maps.
\end{abstract}

\section{RESUMEN}

Un espacio $X$ se llama débilmente fuertemente estrellaMenger si para cada sucesión $\left(\mathcal{U}_{n}: n \in \omega\right)$ de cubrimientos abiertos de $X$, existe una sucesión $\left(F_{n}: n \in \omega\right)$ de subconjuntos finitos de $X$ tales que $\overline{\bigcup_{n \in \omega} S t\left(F_{n}, \mathcal{U}_{n}\right)}$ es $X$. En este artículo, investigamos la relación entre espacios débilmente fuertemente estrella-Menger con otros espacios relacionados. Se muestra que un $P$-espacio Hausdorff paracompacto débilmente estrella Menger es estrella-Menger. También estudiamos las imágenes y preimágenes de espacios débilmente fuertemente estrella-Menger bajo diversos tipos de aplicaciones.

Keywords and Phrases: Stronlgy star-Menger, star-Menger, almost star-Menger, Weakly star-Menger, covering topological spaces.

2020 AMS Mathematics Subject Classification: 54C10, 54D20, 54G10.

\section{(cc) BY-NC}




\section{Introduction}

The study of selection principles in topology and their relations to game theory and Ramsey theory was started by Scheepers [24] (see also [12]). In the last two decades, these have gained enough importance to become one of the most active areas of set theoretic topology. Several covering properties are defined based on these selection principles $([17,18])$. A number of results in the literature show that many topological properties can be described and characterized in terms of star covering properties $([7,21,22])$. The method of stars has been used to study the problem of metrization of topological spaces, and for definitions of several important classical topological notions.

Let us recall that a space $X$ is countably compact (CC) if every countable open cover of $X$ has a finite subcover. Fleischman [10] defined a space $X$ to be starcompact if for every open cover $\mathcal{U}$ of $X$, there exists a finite subset $F$ of $X$ such that $S t(F, \mathcal{U})=X$, where $S t(F, \mathcal{U})=\bigcup\{U \in \mathcal{U}: U \cap F \neq \phi\}$. He proved that every countably compact space is starcompact. Van Douwen in [7] showed that every $T_{2}$ starcompact space is countably compact, but this does not hold for $T_{1}$-spaces (see $[26$, Example 2.5]).

Matveev [20] defined a space $X$ to be absolutely countably compact (ACC) if for each open cover $\mathcal{U}$ of $X$ and each dense subset $D$ of $X$, there exists a finite subset $F$ of $D$ such that $\operatorname{St}(F, \mathcal{U})=X$. It is clear that every $T_{2}$-absolutely countably compact space is countably compact.

Kočinac et al. $([1,2,15,16])$, defined a space $X$ to be strongly star-Menger (SSM) if for each sequence $\left(\mathcal{U}_{n}: n \in \omega\right)$ of open covers of $X$, there exists a sequence $\left(F_{n}: n \in \omega\right)$ of finite subsets of $X$ such that $\left\{S t\left(F_{n}, \mathcal{U}_{n}\right): n \in \omega\right\}$ is an open cover of $X$. The SSM property is stronger than the star-Menger (SM) property.

Pansera [23], defined a space $X$ to be weakly star-Menger (WSM) if for each sequence $\left(\mathcal{U}_{n}: n \in \omega\right)$ of open covers of $X$ there is a sequence $\left(\mathcal{V}_{n}: n \in \omega\right)$ with $\mathcal{V}_{n}$ a finite subset of $\mathcal{U}_{n}$ for each $n \in \omega$, and $\overline{\bigcup_{n \in \omega} S t\left(\cup \mathcal{V}_{n}, \mathcal{U}_{n}\right)}=X$. WSM is weaker than the SSM property.

In this paper we introduce a star property which lies between SSM and WSM called weakly strongly star-Menger (WSSM).

The paper is organized as follows. Section 2 contains some preliminaries used in the paper. In Section 3 we investigate the relationship of WSSM spaces with other related spaces. Section 4 contains the information on subspaces and product spaces of WSSM and in the last Section 5 we study the image and preimage of WSSM spaces under continuous maps. 


\section{Preliminaries}

Throughout this paper a space means topological space. The cardinality of a set $A$ is denoted by $|A|$. Let $\omega$ be the first infinite cardinal and $\omega_{1}$ the first uncountable cardinal, $\mathfrak{c}$ the cardinality of the set of all real numbers. As usual, a cardinal is an initial ordinal and an ordinal is the set of smaller ordinals. Every cardinal is often viewed as a space with the usual order topology. Other terms and symbols that we define follow [9].

We make use of two of the cardinals defined in [8]. Define $\omega^{\omega}$ as the set of all functions from $\omega$ to itself. For all $f, g \in \omega^{\omega}$, we say $f \leq^{*} g$ if and only if $f(n) \leq g(n)$ for all but finitely many $n$. The unbounding number, denoted by $\mathfrak{b}$, is the smallest cardinality of an unbounded subset of $\left(\omega^{\omega}, \leq^{*}\right)$. The dominating number, denoted by $\mathfrak{d}$, is the smallest cardinality of a cofinal subset of $\left(\omega^{\omega}, \leq^{*}\right)$. It is not difficult to show that $\omega_{1} \leq \mathfrak{b} \leq \mathfrak{d} \leq \mathfrak{c}$ and it is known that $\omega_{1}<\mathfrak{b}=\mathfrak{c}, \omega_{1}<\mathfrak{d}=\mathfrak{c}$ and $\omega_{1} \leq \mathfrak{b}<\mathfrak{d}=\mathfrak{c}$ are all consistent with the axioms of ZFC (see [8] for details).

A space $X$ is said to be absolutely strongly star-Menger (ASSM) [6], if for each sequence $\left(\mathcal{U}_{n}: n \in\right.$ $\omega)$ of open covers of $X$ and each dense subset $D$ of $X$, there exists a sequence $\left(F_{n}: n \in \omega\right)$ of finite subsets of $D$ such that $\left\{\operatorname{St}\left(F_{n}, \mathcal{U}_{n}\right): n \in \omega\right\}$ is an open cover of $X$.

A space $X$ is called star-Menger (SM) [15], if for each sequence $\left(\mathcal{U}_{n}: n \in \omega\right)$ of open covers of $X$ there is a sequence $\left(\mathcal{V}_{n}: n \in \omega\right)$ with $\mathcal{V}_{n}$ a finite subset of $\mathcal{U}_{n}$ for each $n \in \omega$, and $\left\{\operatorname{St}\left(\cup \mathcal{V}_{n}, \mathcal{U}_{n}\right)\right.$ : $n \in \omega\}$ is a cover of $X$.

A space $X$ is called almost star-Menger (ASM) [14], if for each sequence $\left(\mathcal{U}_{n}: n \in \omega\right)$ of open covers of $X$ there is a sequence $\left(\mathcal{V}_{n}: n \in \omega\right)$ with $\mathcal{V}_{n}$ a finite subset of $\mathcal{U}_{n}$ for each $n \in \omega$, and $\left\{\overline{\operatorname{St}\left(\cup \mathcal{V}_{n}, \mathcal{U}_{n}\right)}: n \in \omega\right\}$ is a cover of $X$.

Definition 2.1. A space $X$ is called weakly strongly star-Menger (WSSM) if for each sequence $\left(\mathcal{U}_{n}: n \in \omega\right)$ of open covers of $X$, there is a sequence $\left(F_{n}: n \in \omega\right)$ of finite subsets of $X$ such that $\overline{\bigcup_{n \in \omega} S t\left(F_{n}, \mathcal{U}_{n}\right)}=X$.

From the above definitions we have the following diagram of implications:

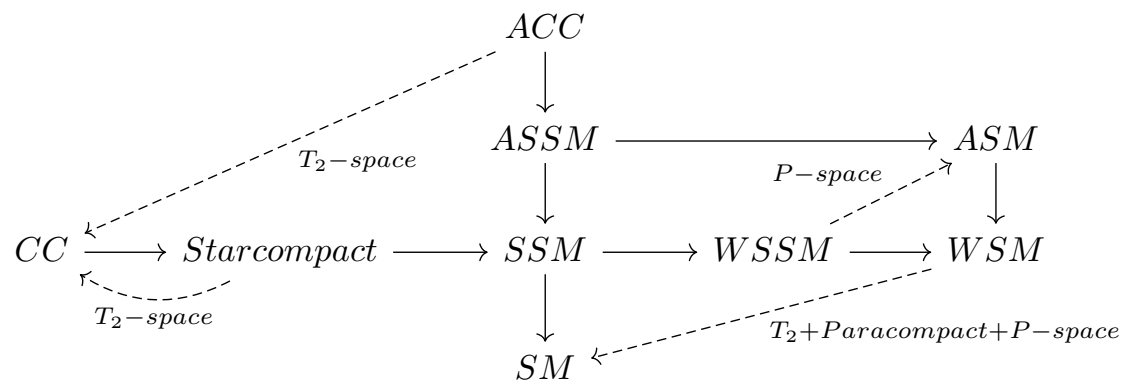

The purpose of this paper is to investigate the relationships of weakly strongly star-Menger spaces with other spaces. In Example 3.3, we have shown that the WSSM property need not be SSM 
property. Presently, we do not know that the WSM property implies WSSM and ASM property. On the other hand, there are several examples in the literature on star-selection principles showing that other reverse implications need not be true.

\section{$3 \quad$ Weakly strongly star-Menger spaces and related spaces}

In this section, we give some results and examples showing relationships of weakly strongly starMenger with other properties.

A subspace (subset) $Y$ of a space $X$ is WSSM if $Y$ is WSSM as a subspace.

Theorem 3.1. If $X$ has a dense WSSM subspace, then $X$ is WSSM.

Proof. If $D=X$ then we are done. Let $D$ be a non-trivial dense WSSM subspace of $X$ and $\left(\mathcal{U}_{n}: n \in \omega\right)$ be a sequence of open covers of $X$. Then $\left(\mathcal{U}_{n}^{\prime}: n \in \omega\right)$ is a sequence of open covers of $D$, where $\mathcal{U}_{n}^{\prime}=\left\{U \cap D: U \in \mathcal{U}_{n}\right\}$. Therefore there exists a sequence $\left(F_{n}^{\prime}: n \in \omega\right)$ of finite subsets of $D$ with $\overline{\bigcup_{n \in \omega} S t\left(F_{n}^{\prime}, \mathcal{U}_{n}^{\prime}\right)}=D$. Hence $\overline{\bigcup_{n \in \omega} S t\left(F_{n}^{\prime}, \mathcal{U}_{n}\right)}=X$ as $D$ is dense in $X$.

Corollary 3.2. Every separable topological space is WSSM.

Given an almost disjoint family $\mathcal{A}$ of infinite subsets of $\omega$ (that is, the intersection of every two distinct elements of $\mathcal{A}$ is finite) the $\psi$-space or the Isbell-Mrówka space associated to $\mathcal{A}$ (denoted by $\psi(\mathcal{A})$ has $\omega \cup \mathcal{A}$ as the underlying set, the points of $\omega$ being isolated, while the basic open neighborhoods of $A \in \mathcal{A}$ are of the form $\{A\} \cup(A \backslash F)$, where $F$ ranges over all finite subsets of $\omega$. For more details (see $[3,11])$.

Example 3.3. There exists a Tychonoff WSSM space X which is not SSM.

Proof. Let $X=\omega \cup \mathcal{A}$ be the Isbell-Mrówka space, where $\mathcal{A}$ is the maximal almost disjoint family of infinite subsets of $\omega$ with $|\mathcal{A}|=\mathfrak{c}$. Then $X$ is not strongly star-Menger ([25, Example 2.3]). But $X$ is WSSM, $\omega$ being a countable dense subset of $X$.

Recall that a topological space $X$ is a $P$-space [13] if every intersection of countably many open subsets of $X$ is open.

Proposition 3.4. A WSSM P-space $X$ is almost star-Menger.

Proof. Let $\left(\mathcal{U}_{n}: n \in \omega\right)$ be sequence of open covers of $X$. Then there exists a sequence $\left(F_{n}\right.$ : $n \in \omega)$ of finite subsets of $X$ with $\overline{\bigcup_{n \in \omega} S t\left(F_{n}, \mathcal{U}_{n}\right)}=X$ as $X$ is WSSM. Since $X$ is $P$-space, $\bigcup_{n \in \omega} \overline{\operatorname{St}\left(F_{n}, \mathcal{U}_{n}\right)}$ is a closed subset of $X$ which contains $\bigcup_{n \in \omega} \operatorname{St}\left(F_{n}, \mathcal{U}_{n}\right)$. Hence $\bigcup_{n \in \omega} \overline{\operatorname{St}\left(F_{n}, \mathcal{U}_{n}\right)}=$ $\overline{\bigcup_{n \in \omega} S t\left(F_{n}, \mathcal{U}_{n}\right)}$. Then we can find a sequence $\mathcal{V}_{n}$ of finite subsets of $\mathcal{U}_{n}$ containing $F_{n}$ such that $\bigcup_{n \in \omega} \overline{\operatorname{St}\left(\cup_{n}, \mathcal{U}_{n}\right)}=X$. 
Theorem 3.5. A Hausdorff paracompact weakly star-Menger P-space $X$ is star-Menger.

Proof. Let $\left(\mathcal{U}_{n}: n \in \omega\right)$ be a sequence of open covers of $X$. Since a Hausdorff paracompact space is regular, for each $x \in X$ there exists an open neighborhood say $V_{n, x}$ of $x$ with $\overline{V_{n, x}} \subseteq U$ for some $U \in \mathcal{U}_{n}$. Let $\mathcal{V}_{n}$ be a locally finite open refinement of the open cover $\left\{V_{n, x}: x \in X\right\}$. Since $X$ is WSM there exists a sequence $\left(\mathcal{V}_{n}^{\prime}: n \in \omega\right)$ such that $\mathcal{V}_{n}^{\prime}$ is a finite subset of $\mathcal{V}_{n}$ for each $n \in \omega$ with $\overline{\bigcup_{n \in \omega} S t\left(\cup \mathcal{V}_{n}^{\prime}, \mathcal{V}_{n}\right)}=X$. Now for each $V \in \mathcal{V}_{n}$ there is a $U_{V} \in \mathcal{U}_{n}$ with $\bar{V} \subseteq U_{V}$. Then for each fixed $n \in \omega, \overline{S t\left(\cup \mathcal{V}_{n}^{\prime}, \mathcal{V}_{n}\right)} \subseteq S t\left(\cup \mathcal{U}_{n}^{\prime}, \mathcal{U}_{n}\right)$, where $\mathcal{U}_{n}^{\prime}$ is finite subset of $\mathcal{U}_{n}$ such that for every $V \in \mathcal{V}_{n}^{\prime}$ there is $U \in \mathcal{U}_{n}^{\prime}$ contaning $\bar{V}$. Therefore, $X=\overline{\bigcup_{n \in \omega} \operatorname{St}\left(\cup \mathcal{V}_{n}^{\prime}, \mathcal{V}_{n}\right)}=\bigcup_{n \in \omega} \overline{S t\left(\cup \mathcal{V}_{n}^{\prime}, \mathcal{V}_{n}\right)}=$ $\bigcup_{n \in \omega} S t\left(\cup \mathcal{U}_{n}^{\prime}, \mathcal{U}_{n}\right)$, because $X$ is a $P$-space.

In [15], Kočinac has shown that the property strongly star Menger is equivalent to the property star-Menger in Hausdorff paracompact space $X$. Then we have the following corollary:

Corollary 3.6. For a Hausdorff paracompact $P$-space $X$, the following statements are equivalent:

(1) $X$ is strongly star-Menger;

(2) $X$ is weak strongly star-Menger;

(3) $X$ is almost star-Menger;

(4) $X$ is weakly star-Menger;

(5) $X$ is star-Menger.

In [13], Kocev defined $d$-paracompact space. A space $X$ is said to be $d$-paracompact if every dense family of subsets of $X$ has a locally finite refinement.

Theorem 3.7. A WSM and d-paracompact space $X$ is almost star-Menger.

Proof. Let $\left(\mathcal{U}_{n}: n \in \omega\right)$ be a sequence of open covers of $X$. As $X$ is WSM, there exists a sequence $\left(\mathcal{V}_{n}: n \in \omega\right)$, where $\mathcal{V}_{n}$ is a finite subset of $\mathcal{U}_{n}$ with $\bigcup\left\{S t\left(\cup \mathcal{V}_{n}, \mathcal{U}_{n}\right): n \in \omega\right\}$ dense in $X$. By the assumption $\left\{S t\left(\cup \mathcal{V}_{n}, \mathcal{U}_{n}\right): n \in \omega\right\}$ has a locally finite refinement say, $\mathcal{W}$. Then $\cup \mathcal{W}=\bigcup_{n \in \omega} S t\left(\cup \mathcal{V}_{n}, \mathcal{U}_{n}\right)$ and therefore $\overline{\cup \mathcal{W}}=\overline{\bigcup_{n \in \omega} S t\left(\cup \mathcal{V}_{n}, \mathcal{U}_{n}\right)}$. As $\mathcal{W}$ is a locally finite family, we have $\overline{\cup \mathcal{W}}=\bigcup_{W \in \mathcal{W}} \bar{W}$. Since each $W \in \mathcal{W}$ is contained in $\operatorname{St}\left(\cup \mathcal{V}_{n}, \mathcal{U}_{n}\right)$ for some $n \in \omega$, $\bigcup_{n \in \omega} \overline{\operatorname{St}\left(\cup_{n}, \mathcal{U}_{n}\right)}=X$.

Corollary 3.8. For a Hausdorff paracompact d-paracompact space $X$, the following statements are equivalent:

(1) $X$ is strongly star-Menger;

(2) $X$ is weak strongly star-Menger; 
(3) $X$ is weakly star-Menger;

(4) $X$ is almost star-Menger;

(5) $X$ is star-Menger.

At the end of this section, we study the relation of WSSM property to Lindelöf covering properties.

Recall, a space $X$ is called Lindelöf if for each open cover $\mathcal{U}$ of $X$ there is countable subset $\mathcal{V}$ of $\mathcal{U}$ such that $X=\bigcup \mathcal{V}$.

Let $X$ be a space of the Example 4.1, then $X$ is WSSM space but it is not Lindelöf, because $X$ has a uncountable discrete closed subset. That means WSSM property does not imply Lindelöf property.

Theorem 3.9. Every $T_{2}$-paracompact WSSM space is Lindelöf.

Proof. Let $\mathcal{U}$ be an open cover of $X$. For each $x \in X$ there exists an open neighborhood say $V_{x}$ of $x$ such that $\overline{V_{x}} \subseteq U$ for some $U \in \mathcal{U}$, because a $T_{2}$-paracompact space is regular. Let $\mathcal{V}$ be a locally finite open refinement of the cover $\left\{V_{x}: x \in X\right\}$. Then $\left(\mathcal{V}_{n}: n \in \omega\right)$ be a sequence of open covers of $X$, where $\mathcal{V}_{n}=\mathcal{V}$ for each $n \in \omega$. Since $X$ is WSSM, there exists a sequence $\left(F_{n}: n \in \omega\right)$ of finite subsets of $X$ such that $\overline{\bigcup_{n \in \omega} S t\left(F_{n}, \mathcal{V}_{n}\right)}=X$. Since $\mathcal{V}_{n}$ is locally finite family, there exist finite subset $\mathcal{V}_{n}^{\prime}$ of $\mathcal{V}_{n}$ such that $S t\left(F_{n}, \mathcal{V}_{n}\right) \subset \cup \mathcal{V}_{n}^{\prime}$, so $X=\overline{\bigcup_{n \in \omega} S t\left(F_{n}, \mathcal{V}_{n}\right)} \subset \overline{\bigcup_{n \in \omega} \cup\left\{V^{\prime}: V^{\prime} \in \mathcal{V}_{n}^{\prime}\right\}}=$ $\bigcup_{n \in \omega} \overline{\cup\left\{V^{\prime}: V^{\prime} \in \mathcal{V}_{n}^{\prime}\right\}}=\bigcup_{n \in \omega} \cup\left\{\overline{V^{\prime}}: V^{\prime} \in \mathcal{V}_{n}^{\prime}\right\}$. For each $V \in \mathcal{V}_{n}$ there is a $U_{V} \in \mathcal{U}$ with $\bar{V} \subseteq U_{V}$. Hence we can constuct a countable subset $\mathcal{U}^{\prime}$ of $\mathcal{U}$ such that $\bigcup_{n \in \omega} \cup\left\{\overline{V^{\prime}}: V^{\prime} \in \mathcal{V}_{n}^{\prime}\right\} \subset \cup \mathcal{U}^{\prime}$.

Definition 3.10. [7] A space $X$ is called strongly star-Lindelöf (in short, SSL) if for each open cover $\mathcal{U}$ of $X$ there is a countable subset $F$ of $X$ such that $S t(F, \mathcal{U})=X$.

Clearly, SSM property implies SSL property. But next we will show that WSSM property need not be SSL property.

A space $X$ is almost star countable [28], if for each open cover $\mathcal{U}$ of $X$ there exists a countable subset $F$ of $X$ such that $\bigcup_{x \in F} \overline{S t(x, \mathcal{U})}=X$.

Evidently, strongly star-Lindelöf $\Rightarrow$ almost star-countable.

Example 3.11. A WSSM space need not be SSL.

Proof. Let $D$ be a discrete space of cardinality $\omega_{1}, X=(\beta D \times(\omega+1)) \backslash((\beta D \backslash D) \times\{\omega\})$ is a subspace of the product space $\beta D \times(\omega+1)$. Then $X$ is WSSM by Lemma 4.4., because $\beta D \times \omega$ is a dense $\sigma$-compact (hence, $\sigma$-countably compact) subset of $X$. But $X$ is not SSL, because $X$ is not almost star countable (see [28, Example 2.5]). 
Theorem 3.12. Every $T_{2}$-paracompact WSSM space is SSL.

Proof. The proof follows the same constructions of Theorem 3.9, thus omitted.

\section{Subspaces and product spaces}

In this section we study subspaces of a WSSM space and also show that product of two WSSM spaces need not be WSSM. For some relative version of star selection principles see $([4,5,19])$.

Example 4.1. A closed subset of WSSM space need not be WSSM.

Proof. Let $\mathbb{R}$ be the set of real numbers, $\mathbb{I}$ the set of irrational numbers and $\mathbb{Q}$ the set of rational numbers. For each irrational $x$ we choose a sequence $\left\{x_{i}: i \in \omega\right\}$ of rational numbers converging to $x$ in the Euclidean topology. The rational sequence topology $\tau$ (see [29, Example 65]) is then defined by declaring each rational open and selecting the sets $U_{n}(x)=\left\{x_{n, i}: i \in \omega\right\} \cup\{x\}$ as a basis for the irrational point $x$. Then the set of irrational points $\mathbb{I}$ is a closed subset of $(\mathbb{R}, \tau)$ and $\mathbb{I}$ as a subspace of the space $(\mathbb{R}, \tau)$ is not WSSM, because it is uncountable discrete subspace. On the other hand, $(\mathbb{R}, \tau)$ is WSSM, because $\mathbb{Q}$ is dense in $(\mathbb{R}, \tau)$.

Proposition 4.2. Every clopen subset of a WSSM space is WSSM.

Proof. Let $Y$ be a clopen subset of a WSSM space $X$ and let $\left(\mathcal{U}_{n}: n \in \omega\right)$ be a sequence of open covers of $Y$. Then $\left(\mathcal{V}_{n}: n \in \omega\right)$, where $\mathcal{V}_{n}=\mathcal{U}_{n} \cup\{X \backslash Y\}$ is a sequence of open covers of $X$. Since $X$ is WSSM, there exists a sequence of finite subsets $F_{n}$ of $X$ with $\overline{\bigcup_{n \in \omega} \operatorname{St}\left(F_{n}, \mathcal{V}_{n}\right)}=X$. Put $F_{n}^{\prime}=Y \cap F_{n}$. Then clearly, $\overline{\bigcup_{n \in \omega} S t\left(F_{n}^{\prime}, \mathcal{U}_{n}\right)}=Y$.

Song [25] gave an example showing that the product of two countably compact spaces is not strongly star compact. This example also shows that the product of two WSSM spaces need not be WSSM. We sketch it below.

Example 4.3. There exist two countably compact (and hence WSSM) spaces $X$ and $Y$ such that $X \times Y$ is not WSSM.

Proof. Let $D$ be the discrete space of the cardinality c. We define $X=\bigcup_{\alpha<\omega_{1}} E_{\alpha}, Y=\bigcup_{\alpha<\omega_{1}} F_{\alpha}$, where $E_{\alpha}$ and $F_{\alpha}$ are the subsets of $\beta(D)$ which are defined inductively so as to satisfy the following three conditions:

(1) $E_{\alpha} \cap F_{\beta}=D$ if $\alpha \neq \beta$.

(2) $\left|E_{\alpha}\right| \leq \mathfrak{c}$ and $\left|F_{\alpha}\right| \leq \mathfrak{c}$.

(3) every infinite subset of $E_{\alpha}\left(\right.$ resp., $\left.F_{\alpha}\right)$ has an accumulation point in $E_{\alpha+1}$ (resp, $\left.F_{\alpha+1}\right)$. 
These sets $E_{\alpha}$ and $F_{\alpha}$ are well-defined since every infinite closed set in $\beta(D)$ has the cardinality $2^{\mathfrak{c}}$, for detail see [30]. Then, $X \times Y$ is not WSSM since the diagonal $\{\langle d, d\rangle: d \in D\}$ is a discrete open and closed subset of $X \times Y$ with the cardinality $\mathfrak{c}$ and the property WSSM is preserved by open and closed subsets. Hence product of WSSM spaces need not be WSSM.

Now we give some positive results.

Recall that a subset $A$ of a space $X$ is said to be $\sigma$-countably compact if it is union of countably many countably compact subset of $X$.

Lemma 4.4. If a space $X$ has a $\sigma$-countably compact dense subset, then $X$ is WSSM.

Proof. Let $D=\bigcup_{n \in \omega} D_{n}$ be a dense subset of $X$, where each $D_{n}$ is countably compact subset of $X$. Let $\left(\mathcal{U}_{n}: n \in \omega\right)$ be a sequence of open covers of $X$. Then for each $n \in \omega$ there exists a finite subset say $F_{n}$ of $D_{n}$ such that $D_{n} \subset S t\left(F_{n}, \mathcal{U}_{n}\right)$. So $\left(F_{n}: n \in \omega\right)$ is a sequence of finite subsets of $D$ such that $D=\bigcup_{n \in \omega} \operatorname{St}\left(F_{n}, \mathcal{U}_{n}\right)$.

Theorem 4.5. Let $\left\{X_{n}: n \in \omega\right\}$ be a countable collection of countably compact subsets of space $X$ such that $X=\bigcup_{n \in \omega} X_{n}$. Then $X$ is a WSSM space.

Corollary 4.6. If $\left\{X_{n}: n \in \omega\right\}$ is a countable collection of mutually disjoint countably compact spaces, then the topological sum $\bigoplus_{n \in \omega} X_{n}$ is WSSM if and only if each $X_{n}$ is WSSM.

Corollary 4.7. If $X$ is countably compact space, then $X \times \omega$, where $\omega$ has discrete topology is WSSM.

Proof. Since $X \times \omega$ is homeomorphic to $\bigoplus_{n \in \omega} X \times\{n\}$ and $X \times\{n\}$ is homeomorphic to $X$ for each $n \in \omega$. Then, by Corollary 4.6, $\bigoplus_{n \in \omega} X \times\{n\}$ is WSSM.

\section{$5 \quad$ Images and Preimages}

In this section we study the images and preimages of WSSM spaces under continuous maps.

Theorem 5.1. A continuous image of a WSSM space is WSSM.

Proof. Let $f: X \rightarrow Y$ be a continuous surjection and $X$ be a WSSM space. Let $\left(\mathcal{U}_{n}: n \in \omega\right)$ be sequence of open covers of $Y$. Then $\left(\mathcal{U}_{n}^{\prime}: n \in \omega\right)$, where $\mathcal{U}_{n}^{\prime}=\left\{f^{-1}(U): U \in \mathcal{U}_{n}\right\}$ is a sequence of open covers of $X$. Thus there exists a sequence $\left(F_{n}^{\prime}: n \in \omega\right)$ of finite subsets of $X$ such that $\overline{\bigcup_{n \in \omega} S t\left(F_{n}^{\prime}, \mathcal{U}_{n}^{\prime}\right)}=X$. Let $F_{n}=f\left(F_{n}^{\prime}\right)$. Then $\left(F_{n}: n \in \omega\right)$ is a sequence of finite subsets of $Y$. Hence result follows from the fact that for an arbitrary $y \in Y$ and each neighbourhood $U$ of $y$, $U \cap \bigcup_{n \in \omega} S t\left(F_{n}, \mathcal{U}_{n}\right) \neq \phi$. 
Next we turn to consider preimages. We show that the preimage of a WSSM space under a closed 2-to-1 continuous map need not be WSSM. First we discuss examples.

Recall the Alexandroff duplicate $A(X)$ of a space $X$. The underlying set $A(X)$ is $X \times\{0,1\}$; each point of $X \times\{1\}$ is isolated and a basic neighborhood of $<x, 0>\in X \times\{0\}$ is a set of the form $(U \times\{0\}) \cup((U \times\{1\}) \backslash\{<x, 1>\})$, where $U$ is a neighborhood of $x$ in $X$.

Example 5.2. Assuming $\mathfrak{d}=\mathfrak{c}$, there exists a WSSM space $X$ such that $A(X)$ is not WSSM.

Proof. Assume that $\mathfrak{d}=\mathfrak{c}$. Let $X=\omega \cup \mathcal{A}$ be the Isbell-Mrówka space with $|\mathcal{A}|=\omega_{1}$. Then $X$ is absolutely strongly star-Menger ([27, Example 3.5]), and hence WSSM. However $A(X)$ is not WSSM. Since the set $\mathcal{A} \times\{1\}$ is an open and closed subset of $A(X)$ with $|\mathcal{A} \times\{1\}|=\omega_{1}$, and for each $a \in \mathcal{A}$, the point $\langle a, 1\rangle$ is isolated in $A(X)$. Hence $A(X)$ is not WSSM, since every open and closed subset of a WSSM space is WSSM, and $\mathcal{A} \times\{1\}$ is not WSSM .

Example 5.3. Assuming $\mathfrak{d}=\mathfrak{c}$, there exists a closed 2-to-1 continuous map $f: X \rightarrow Y$ such that $Y$ is a WSSM space, but $X$ is not a WSSM.

Proof. Let $Y$ be the space $X$ of Example 5.2. Then $Y$ is WSSM. Let $X$ be the space $A(Y)$. Then $X$ is not WSSM. Let $f: X \rightarrow Y$ be the projection. Then $f$ is a closed 2-to-1 continuous map, which completes the proof.

Theorem 5.4. Let $f$ be an open and closed, finite-to-one continuous map from a space $X$ onto a WSSM space $Y$. Then $X$ is WSSM.

Proof. Let $\left(\mathcal{U}_{n}: n \in \omega\right)$ be a sequence of open covers of $X$ and let $y \in Y$. Since $f^{-1}(y)$ is finite, for each $n \in \omega$ there exists a finite sub-collection $\mathcal{U}_{n_{y}}$ of $\mathcal{U}_{n}$ such that $f^{-1}(y) \subset \cup \mathcal{U}_{n_{y}}$ and $U \cap f^{-1}(y) \neq \phi$ for each $U \in \mathcal{U}_{n_{y}}$. Since $f$ is closed, there exists an open neighbourhood $V_{n_{y}}$ of $y$ in $Y$ such that $f^{-1}\left(V_{n_{y}}\right) \subseteq \cup\left\{U: U \in \mathcal{U}_{n_{y}}\right\}$. Since $f$ is open, we can assume that $V_{n_{y}} \subseteq \cap\left\{f(U): U \in \mathcal{U}_{n_{y}}\right\}$. For each $n \in \omega$, take such open set $V_{n_{y}}$ for each $y \in Y$, and put $\mathcal{V}_{n}=\left\{V_{n_{y}}: y \in Y\right\}$ of $Y$. Thus $\left(\mathcal{V}_{n}: n \in \omega\right)$ is a sequence of open covers of $Y$. Since $Y$ is WSSM, there exists a sequence $\left(F_{n}: n \in \omega\right)$ of finite subsets of $Y$ such that $\overline{\bigcup_{n \in \omega} S t\left(F_{n}, \mathcal{V}_{n}\right)}=Y$. Since $f$ is finite to one, the sequence $\left(f^{-1}\left(F_{n}\right): n \in \omega\right)$ is a sequence of finite subsets of $X$. We show that $\overline{\bigcup_{n \in \omega} S t\left(f^{-1}\left(F_{n}\right), \mathcal{U}_{n}\right)}=X$. Let $x \in X$ and $V$ be an arbitrary neighbourhood of $x$ in $X$, then $f(V)$ is a neighbourhood of $y=f(x)$ as $f$ is an open map. Then there exist $n \in \omega$ and $y^{\prime} \in Y$ such that $y \in f(V) \cap V_{n_{y^{\prime}}}$ with $V_{n_{y^{\prime}}} \cap F_{n} \neq \phi$. Choose $U \in \mathcal{U}_{n_{y^{\prime}}}$. Then $V_{n_{y^{\prime}}} \subseteq f(U)$. Hence $U \cap f^{-1}\left(F_{n}\right) \neq \phi$ as $V_{n_{y^{\prime}}} \cap F_{n} \neq \phi$. Therefore, $x \in \overline{\bigcup_{n \in \omega} S t\left(f^{-1}\left(F_{n}\right), \mathcal{U}_{n}\right)}$. This shows that $X$ is WSSM. 


\section{Acknowledgements}

(1) The first author acknowledges the fellowship grant of University Grant Commission, India.

(2) The authors would like to thank referees for their valuable suggestions which led to improvements of the paper in several places. 


\section{References}

[1] M. Bonanzinga, F. Cammaroto and Lj. D. R. Kočinac, "Star-Hurewicz and related properties", Appl. Gen. Topol., vol. 5, no. 1, pp. 79-89, 2004.

[2] M. Bonanzinga, F. Cammaroto, Lj. D. R. Kočinac and M. V. Matveev, "On weaker forms of Menger, Rothberger and Hurewicz properties", Mat. Vesnik, vol 61, no. 1, pp. 13-23, 2019.

[3] M. Bonanzinga and M. V. Matveev, "Some covering properties for $\psi$-spaces", Mat. Vesnik, vol. 61 , no. 1, pp. 3-11, 2009.

[4] M. Bonanzinga, M. V. Matveev and B. A. Pansera, "When can a cover of a product be refined by a product of covers", Question Answers Gen. Topology, vol. 26, no. 2, pp. 67-74, 2008.

[5] M. Bonanzinga and B. A. Pansera, "Relative versions of some star selection principles", Acta Math. Hungar., vol. 117, no. 3, pp. 231-243, 2007.

[6] A. Caserta, G. M. Di Maio and Lj. D. R. Kočinac, "Versions of properties $(a)$ and ( $p p)$ ", Topology Appl., vol. 158, no. 12, pp. 1360-1368, 2011.

[7] E. K. van Douwen, G.K. Reed, A. W. Roscoe and I. J. Tree, "Star covering properties", Topology Appl., vol. 39, no. 1, pp. 71-103, 1991.

[8] E. K. van Douwen, "The integers and topology", in: K. Kunen, J.E. Vaughan (Eds.), Handbook of Set-Theoretic Topology, Amsterdam: North-Holland, pp. 111-167, 1984.

[9] R. Engelking, General Topology, Revised and completed edition, Berlin : Heldermann Verlag, 1989.

[10] W. M. Fleischman, "A new extension of countable compactness", Fund. Math., vol. 67, no. 1, pp. 1-9, 1971.

[11] L. Gillman and M. Jerison, Rings of Continuous Functions, New York: Van Nostrand, 1960.

[12] W. Just, A. W. Miller, M. Scheepers and P. J. Szeptycki, "The combinatorics of open covers II". Topology Appl.. vol. 73, pp. 241-266, 1996.

[13] D. Kocev, "Menger-type covering properties of topological spaces", Filomat, vol. 29, no. 1, pp. 99-106, 2015.

[14] D. Kocev, "Almost Menger and related spaces", Mat. Vesnik, vol. 61, no. 2, pp. 173-180, 2009.

[15] Lj. D. R. Kočinac, "Star-Menger and related spaces", Publ. Math. Debrecen, vol. 55, no. 3-4, pp. 421-431, 1999. 
[16] Lj. D. R. Kočinac, "Star-Menger and related spaces II", Filomat, no. 13, pp. 129-140, 1999.

[17] Lj. D. R. Kočinac, "Star selection principles: a survey", Khayyam J. Math., vol. 1, no.1, pp. 82-106, 2015.

[18] Lj. D. R. Kočinac, "Variations of classical selection principles: An overview", Quaest. Math., vol. 43 (2020), no.8, pp. 1121-1153, 2020.

[19] Lj. D. R Kočinac and C. Guido, "Relative covering properties", Questions Answers Gen. Topology, vol. 19, no. 1, pp. 107-114, 2001.

[20] M. V. Matveev, "Absolutely countably compact spaces", Topology Appl., vol. 58, no.1, pp. 81-92, 1994.

[21] M. V. Matveev, "Properties close to pseudocompactness and countable compactness", Vestnik Moskov. Ser. I Mat. Mekh., no. 2, pp. 24-27, 1984.

[22] M. V. Matveev, "A survey on star covering properties", Topology Atlas (1998), Preprint No. 330.

[23] B. A. Pansera, "Weaker forms of the Menger property", Quaest. Math., vol. 35, no. 2, pp. 161-169, 2013.

[24] M. Scheepers, "Combinatorics of open covers (I): Ramsey theory", Topology Appl., vol. 69, no. 1, pp. 31-62, 1992.

[25] Y.-K. Song, "Remarks on strongly star-Menger spaces", Comment. Math. Univ. Carolin., vol. 54, no. 1, pp. 97-104, 2013.

[26] Y.-K. Song, "On countable star-covering properties", Appl. Gen. Topol., vol. 8, no. 2, pp. $249-258,2007$.

[27] Y.-K. Song, "Absolutely strongly star-Menger spaces", Topology Appl., vol 160, no. 3, pp. $475-481,2013$.

[28] Y.-K. Song, "Some remarks on almost star countable spaces", Studia Sci. Math. Hungar., vol. 52, no. 2, pp. 12-20, 2015.

[29] L. A. Steen and J. A. Seebach, Counterexamples in Topology, New York: Dover Publications, 1995.

[30] R. C. Walker, The Stone-Čech Compactification, Ergebnisse der Mathematik und ihrer Grenzgebiete, Band 83, New York-Berlin: Springer, 1974. 